\title{
Antibiotic Residues: Emerging Food Safety and Public Health Concern
}

\section{ISSN: 2637-8078}

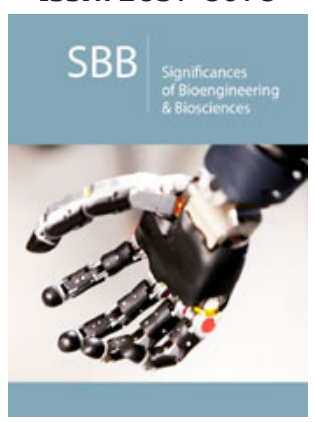

*Corresponding author: Rajkishor Gogoi, Department of Veterinary Pharmacology \& Toxicology, India

Submission: 非 April 22, 2019

Published: 眥April 29, 2019

Volume 3 - Issue 2

How to cite this article: Rajkishor Gogoi Antibiotic Residues: Emerging Food Safety and Public Health Concern.Significances Bioeng Biosci.3(2). SBB.000557.2019. DOI: 10.31031/SBB.2019.03.000557.

Copyright@ Rajkishor Gogoi, This article is distributed under the terms of the Creative Commons Attribution 4.0 International License, which permits unrestricted use and redistribution provided that the original author and source are credited.

\author{
Rajkishor Gogoi* \\ Department of Veterinary Pharmacology \& Toxicology, India
}

\section{Opinion}

The ongoing emergence of antibiotic residues in animal food products is a public health issue of great concern. Antibiotics are widely used both therapeutically and as growth promoters in animal farms. Antibiotics are used for improving growth performance in broilers and fatteners. They may produce improved growth rate due to thinning of mucous membrane of the gut and facilitates better absorption. They alter gut motility to enhance better assimilation. They produce favourable conditions to beneficial microbes in the gut of animal by destroying harmful bacteria and partitioning proteins to muscle accretion by suppressing monokines. Antibiotics also favour growth by decreasing degree of activity of the immune system, reduced waste of nutrients and reduce toxin formation. In most of the cases only young growing animals and poultry are responsive to antibiotic mediated growth promotion. Indiscriminating therapeutic use of antibiotics such as in cases of pyrexia, inflammation, treatment of wounds and viral diseases have wide residual effects on edible tissues. Animals and poultry are receiving sub therapeutic levels of antibiotics to prevent possible infection. Antimicrobials are used either directly or indirectly during the production, processing and storage of milk and milk products. FDA prohibits indiscriminate use of nitrofurazone, sulphonamides, chloramphenicol, furazolidone and flouroquinolones in milk producing animals.

When animals are injected with antibiotics, the drugs accumulate in the body. These accumulated drugs are called antibiotic residue. Antibiotic residue is detectible in the blood, muscle tissue and body fluids, like milk. After the animal is taken off the antibiotics, the drug residue eventually passes out of their system. There are tests to determine the level of antibiotic residue present in the animal's system. Techniques mainly used for the screening and determination of antibiotic residues in animal food products are mainly ELISA, HPLC, Liquid chromatography, Gas chromatography, Paper chromatography. High residue levels will signal the farmer to withhold that animal's product from the market for a longer period of time. All food animals that are injected with antibiotics must go through a withdrawal period before the meat or milk can be shipped to the market. A withdrawal period is the amount of time it takes any antibiotic residue to be flushed out of the animal's system, enough that it will not show up on standard tests. Antibiotics administered by oral route are slowly absorbed and excreted with faeces. Thus, animal wastes from farms contain antibiotics in active form. Very often wastes from terrestrial animals are used for field manuring. Antibiotics are environmental contaminants as they are biologically active. Antibacterial agents are usually very soluble. In order to be as effective as possible they often have a low biodegradability. This property poses a potential for bioaccumulation and persistence in the environment. Drugs, which have specific mode of actions, may probably exert effects on terrestrial and aquatic ecosystems, when released into the environment. Antibiotic residues may cause different complications, of which antibiotic sensitivity, allergic reaction, reproductive and endocrine disruption are of greater concern. Transfer of antibiotic resistant bacteria to the human, Immunopathological effects, Autoimmunity, Carcinogenicity, Mutagenicity, Nephropathy, Hepatotoxicity, Reproductive disorders, Bone marrow toxicity, Allergy are some of the important toxicities produced due to antibiotic residues in animal food products like milk, meat and eggs. Steps may be undertaken for antibiotic residue prevention by making individuals and organizations 
aware of the problem through education by veterinary personnel, organizations and governmental agencies.

Use of activated charcoal, resin and UV irradiation also help for antibiotic inactivation. Rapid screening procedures for the analysis of antibiotic residues and instant grading and prohibition of food containing antibiotics. Residues are also degraded by cooking methods like microwaving, boiling, steaming, frying etc. and inactivation of antibiotics by processing of milk. Storage and freezing also to some extent decreases residue level in our food. Refrigeration causes disappearance of penicillin. In pasteurization most of antibiotics loses activity. Irrational use of antibiotics in field veterinary practices should be avoided. Development of simple and economic field test to identify drug residue in edible animal products must be initiated. Nationwide monitoring and periodic surveillance of microbial residue in edible tissues and milk and establishment of MRL for antibiotic residues in animal products is the need of the hour.

For possible submissions Click below: 